determine whether the test had affected the diagnostic outcome directly, indirectly or not at all. 78 (81\%) of the tests resulted in direct outcomes, $12(13 \%)$ resulted in indirect outcomes and $6(6 \%)$ had no impact on further investigation or diagnosis. A significant proportion (27\%) of patients undergoing CPET for UD responded normally to exercise and were discharged with reassurance. $15 \%$ were diagnosed with dysfunctional breathing and referred to a specialist physiotherapist for breathing retraining. 57 (38\%) patients were diagnosed as having an exclusively normal or dysfunctional breathing response to exercise. Of these, many had undergone investigation prior to CPET including 19 ECG, $14 \mathrm{~V} / \mathrm{Q}$ scans, nine CT scans, six coronary angiograms, four cardiac treadmill tests, two nuclear medicine stress tests and one cardiac MRI.

Conclusions By introducing CPET at an earlier stage in the diagnostic algorithm for UD, many invasive and expensive investigations could have been avoided. CPET resulted in direct outcomes for $81 \%$ of the patients, highlighting the diagnostic importance of CPET in the investigation of UD.

\section{P190 LONG-TERM OUTCOME OF BRONCHIAL ARTERY EMBOLISATION (BAE) FOR MASSIVE HAEMOPTYSIS}

doi:10.1136/thoraxjnl-2011-201054c.190

A Adlakha, R Rupta, A Sebastian, P Tait, J Jackson, P Ind. Hammersmith Hospital, London, UK

Background BAE for massive haemoptysis is potentially life-saving with low short-to-medium term failure rates in previous studies. We aimed to characterise patients referred for BAE, to examine long-term treatment success and identify risk factors for requiring repeat BAE. Methods We retrospectively identified all patients undergoing BAE from 1994 to 2007. We collated data from hospital databases and primary care on demographics, respiratory diagnoses and procedure with follow-up of up to 16 years. Outcomes were all-cause mortality and recurrence of haemoptysis requiring repeat BAE.

Results 158 patients were embolised on 208 occasions. 85 (54\%) patients were male and median age was 54 (IOR: 41-67)y. The most common underlying diagnoses were aspergilloma $(n=38 ; 24 \%$ of patients), bronchiectasis ( $n=24 ; 15 \%)$, unidentified cause $(n=17$; $11 \%$ ) chronic tuberculosis ( $n=14 ; 9 \%)$, active tuberculosis $(n=12$; $8 \%$ ) and cystic fibrosis $(n=11 ; 7 \%)$. All-cause mortality at 1 month and 3 years was $5.3 \%$ and $29.7 \%$, and need for repeat BAE was $4.7 \%$ and $30.7 \%$ respectively. Repeat BAE at 3 years was most common with aspergilloma (50\%) and least common with active TB (0\%). 3year mortality was highest in cystic fibrosis (40\%) and least with unknown cause $(7.7 \%)$ Neither number nor location of vessels embolised predicted mortality or need for repeat BAE. No major procedural complications were noted.

Conclusions BAE by experienced operators is a safe, minimally invasive procedure for massive haemoptysis with excellent shortterm success. It does not prevent late recurrence of haemoptysis nor obviate the need for repeat BAE, the risk of which is related to the underlying disease rather than to technical aspects of the procedure.

\section{Lung cancer: clinical studies}

P191 A META-ANALYSIS OF ADJUSTED AND UNADJUSTED
OBSERVATIONAL STUDIES OF SLEEVE LOBECTOMY VS
PNEUMONECTOMY FOR NON-SMALL CELL LUNG CANCER

doi:10.1136/thoraxjnl-2011-201054c.191

H Yamamoto, H Takagi, S Goto, M Matsui, T Umemoto. Department of Cardiothoracic Surgery, Shizuoka Medical Cente, Shizuoka, Japan

Introduction and Objectives A previous (published in 2007) metaanalysis of unadjusted results from observational studies suggested that sleeve lobectomy offered better long-term survival than did pneumonectomy for non-small cell lung cancer (NSCLC). Since the meta-analysis was conducted, a number of studies, which included ones providing adjusted mortality data, have been published to date. We performed an updated meta-analysis of sleeve lobectomy vs pneumonectomy for long-term mortality in NSCLC, combining separately adjusted and unadjusted results.

Methods The MEDLINE and EMBASE databases and the Cochrane Library and Central Register of Controlled Trials were searched using PubMed and OVID. Studies considered for inclusion met the following criteria: the design was a study comparing sleeve lobectomy vs pneumonectomy; the study population was patients with NSCLC; and main outcomes included long-term all-cause mortality. From each individual study, hazard ratios (HRs) for mortality and 95\% CIs were abstracted. Study-specific estimates were combined using inverse variance-weighted averages of logarithmic HRs.

Results Our search identified no randomised trials and 14 observational comparative studies that included 7 ones providing adjusted mortality data. Adjustment methods included matching (with propensity score or tumour location and invasion) and multivariate Cox proportional hazard regression. Separately pooled analysis of seven adjusted (1013 patients) and seven unadjusted studies (2278 patients) demonstrated respectively a statistically significant lower all-cause mortality by $36 \%$ and $33 \%$ associated with sleeve lobectomy relative to pneumonectomy in fixed effects models (adjusted HR, 0.64; 95\% CI 0.53 to 0.77; p $<0.00001$; unadjusted HR, 0.67; $95 \%$ CI 0.58 to $0.77 ; p<0.00001$; Abstract P191 figure 1). There was minimal study heterogeneity and accordingly little difference in the pooled result from random-effects modelling. When data from all the 14 studies (3291 patients) were pooled using a fixed-effects model, sleeve lobectomy was associated with lower all-cause mortality by $34 \%$ relative to pneumonectomy that remained statistically significant ( $\mathrm{HR}, 0.66$; $95 \%$ CI 0.59 to $0.74 ; \mathrm{p}<0.00001$ ).

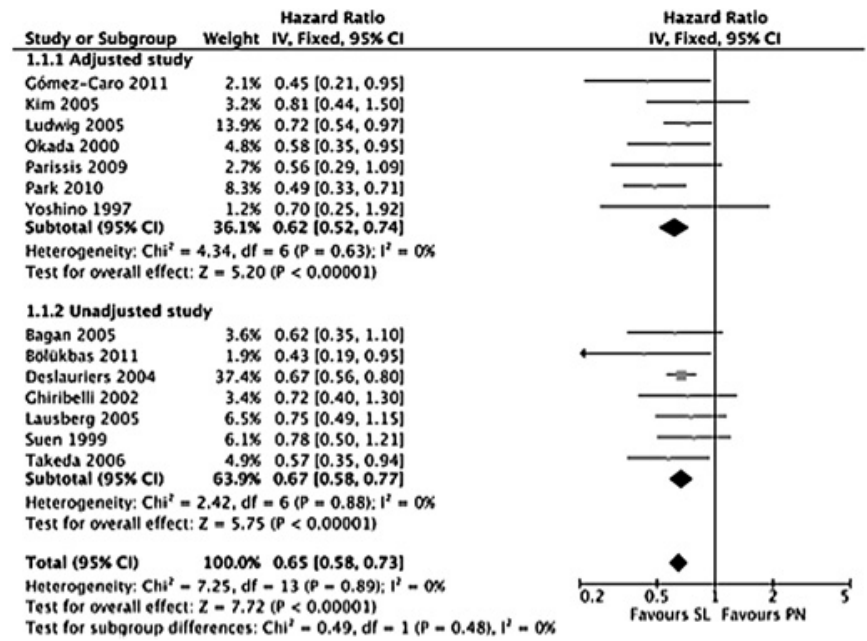

Abstract P191 Figure 1 Long term all-cause mortality patients with non-small cell lung cancer assigned to sleeve lobectomy (SL) vs pneumonectomy (PN).

Conclusions Sleeve lobectomy is likely to have a benefit for longterm all-cause mortality over pneumonectomy in NSCLC. Sleeve lobectomy rather than pneumonectomy should be considered for anatomically suited NSCLC.

\section{P192 SHORT-TERM OUTCOME OF ATTEMPTED CURATIVE RESECTION FOR LUNG CANCER IN ELDERLY PATIENTS}

doi:10.1136/thoraxjnl-2011-201054c.192

A Nazir, S M Kazmi, D Nazareth, J Greenwood, M Ledson, M Walshaw. Liverpool Hear and Chest Hospital NHS Foundation Trust, Liverpool, UK

Background Although resection offers the best chance of cure for most solid tumours, including the lung, $<2 \%$ of elderly patients 